\title{
The INCA System: A Further Step Towards a Telemedical Artificial Pancreas
}

\author{
Enrique J. Gómez, Senior Member, IEEE, M. Elena Hernando Pérez, Member, IEEE, Thomas Vering, \\ Mercedes Rigla Cros, Oliver Bott, Gema García-Sáez, Peter Pretschner, Eulalia Brugués, Oliver Schnell, \\ Caroline Patte, Joachim Bergmann, Ralf Dudde, and Alberto de Leiva
}

\begin{abstract}
Biomedical engineering research efforts have accomplished another level of a "technological solution" for diabetes: an artificial pancreas to be used by patients and supervised by healthcare professionals at any time and place. Reliability of continuous glucose monitoring, availability of real-time programmable insulin pumps, and validation of safe and efficient control algorithms are critical components for achieving that goal. Nevertheless, the development and integration of these new technologies within a telemedicine system can be the basis of a future artificial pancreas. This paper introduces the concept, design, and evaluation of the "intelligent control assistant for diabetes, INCA" system. INCA is a personal digital assistant (PDA)-based personal smart assistant to provide patients with closed-loop control strategies (personal and remote loop), based on a real-time continuous glucose sensor (Guardian RT, Medtronic), an insulin pump (D-TRON, Disetronic Medical Systems), and a mobile general packet radio service (GPRS)-based telemedicine communication system. Patient therapeutic decision making is supervised by doctors through a multiaccess telemedicine central server that provides to diabetics and doctors a Web-based access to continuous glucose monitoring and insulin infusion data. The INCA system has been technically and clinically evaluated in two randomized and crossover clinical trials showing an improvement on glycaemic control of diabetic patients.
\end{abstract}

Index Terms-Artificial pancreas, diabetes, smart assistant, telemedicine.

\section{INTRODUCTION}

D IABETES is a metabolic disorder primarily characterized by elevated blood glucose levels and by microvascular and cardiovascular complications that substantially increase the morbidity and mortality associated with the disease, and reduce the quality of life of diabetic patients. The cause of diabetes continues to be unknown, although both genetics and environmental

Manuscript received December 1, 2006; revised May 30, 2007. This work was supported in part by the 5th European Union Framework Programme under Grant IST-2001-37632, in part by the Switzerland's Federal Office for Education and Science, and in part by the Spanish Field-Initiated Studies (FIS) Grant "Public Archives and Records Infrastructure Support (PARIS)"- FIS PI04246.

E. J. Gómez, M. E. Hernando Pérez, and G. García-Sáez are with the Universidad Politécnica de Madrid, 28040-Madrid, Spain (e-mail: egomez@gbt.tfo.upm.es).

T. Vering and C. Patte are with the Disetronic Medical Systems AG, 3401 Burgdorf, Switzerland.

M. R. Cros, E. Brugués, and A. de Leiva are with the Endocrinology Department, Hospital Sant Pau, 08025 Barcelona, Spain.

O. Bott, P. Pretschner, and J. Bergmann are with the Technische Universität Braunschweig, 38106 Braunschweig, Germany

O. Schnell is with Diabetes Research Center, Department of Cardiovascular Disease, Ludwig-Maximilians-University, 80804 Munich, Germany.

R. Dudde is with the Fraunhofer Institut für Siliziumtechnologie, 25524 Itzehoe, Germany.

Digital Object Identifier 10.1109/TITB.2007.902162 factors appear to play important roles. At least 150 million people today are diagnosed with diabetes, and by 2025, this number is expected to rise to 300 million [1].

The vast majority of diabetes cases fall into broad etiopathogenetic categories: Type 1, Type 2, gestational diabetes, and others. In Type 1 diabetes, the cause is an absolute deficiency of insulin secretion. Nowadays, a well-treated Type 1 diabetic patient can expect to have an almost normal life span as the benefits of intensive therapy management have been well established, and include reduced long-term complications [2]. Many different technologies, methods, and approaches have been developed to improve the diagnosis, monitoring, and treatment of diabetic patients such as biomedical sensors, as a basis for continuous blood glucose monitors; biomedical instrumentation, for self-monitoring data collection and continuous insulin delivery; mathematical modeling, for better understanding of the physiological processes involved in the metabolic control [3]; and application of information technologies, such as database management systems, data analysis and visualization tools, intelligent decision support techniques, and telemedicine systems. Comprehensive reviews on biomedical engineering technologies applied to diabetes management have been published [4], [5].

The combination of insulin pumps and continuous glucose monitoring systems seems to be the best near future solution to achieve a good metabolic control for insulin-treated diabetic patients. A closed-loop device capable of maintaining normoglycaemia over extended periods of time could dramatically improve the quality of metabolic control of insulin-treated diabetic patients [6]. Although the intravenous route is ideal for control, the implementation of an ambulatory closed-loop system imposes the use of the subcutaneous (SC) route as the least invasive solution for insulin delivery and blood glucose measurement. However, the glycaemic control based on the SC route is complex because of the delayed exogenous insulin absorption and action during SC infusion and the delays due to glucose monitoring systems. Several closed-loop control algorithms for a subcutaneous infusion versus subcutaneous sensing (SC-SC) setup have been reported [7], [8].

The dream of an "artificial pancreas" is starting to become reality [9], [10], supported by the increased availability of continuous glucose monitoring solutions, the integration of control systems capable of closing the loop by modifying the pump parameters, and the development of portable devices with capabilities to manage the former technologies [11]. One of the most advanced solutions to achieve an SC-SC closed-loop control system has been developed within the 
research project "advanced insulin infusion using a control loop" (ADICOL) [12] that developed a novel nonlinear model predictive glucose controller, running on a portable hand-held device to administer automatically a precise insulin dosage using the information provided by the glucose sensor.

Telemedicine is providing innovative solutions in the effective treatment of diabetic patients [13]-[15]. A telemedicine system enhances the possibilities of traditional clinical procedures, providing both doctors and patients with an integrated service to manage and improve daily diabetes care. Some of those benefits are: 1) the increase of the quality and quantity of the information collected by patients, affording a better decision-making process for doctors and patients; 2) the improvement in the number of therapy adjustments to be performed by doctors; 3 ) a better physician-patient communication procedure; and 4) the impact of the system to enhance the metabolic control of patients.

The number of telemedicine experiences for diabetes care has significantly increased lately. Some of those experiences have been focused on specific technological solutions for diabetes management such as web-based systems [16] or multiaccess services [17], while others are oriented toward aspects of the illness such as depression [18] or diabetic retinopathy [19].

This paper presents a further step toward the artificial pancreas through the proof of concept of what we call a "telemedical artificial pancreas." The research has been performed by a research consortium funded by the European Union (EU) project "INCA-intelligent control assistant for diabetes." The paper describes the INCA concept, the design of the mobile telemedicine closed-loop architecture, and presents the preliminary clinical evaluation results. Beyond that, a validated simulation for the systematic analysis of the diabetes treatment workflow, use-cases, and cost-benefit estimations has been accomplished.

\section{INCA CONCEPT}

The INCA telemedical artificial pancreas is built on two interlinked loops (see Fig. 1): the personal loop that allows the wireless communication between a PDA-based smart assistant (SA), an insulin pump, and a continuous glucose measurement device; and the remote loop that connects the diabetic patient to the health care professional via his/her SA and its wireless connection to a telemedicine central server (TMCS).

The INCA concept implies four control strategies; each of them is supported by a special setup of the SA.

1) Patient control: The patient decides the changes in the insulin pump programming by using the information coming from a glucose sensor. The process is supervised a posteriori by doctors based on the telemedicine services.

2) Doctor control: The health care professional suggests changes in the insulin therapy using the continuous glucose and insulin infusion information coming from the personal SA through the TMCS.

3) Remote loop control algorithms: The SA is remotely programmed by automatic control procedures implemented in the telemedicine server, under doctors' supervision.

4) Personal loop control algorithms: The closed-loop algorithms implemented in the SA provide a real-time

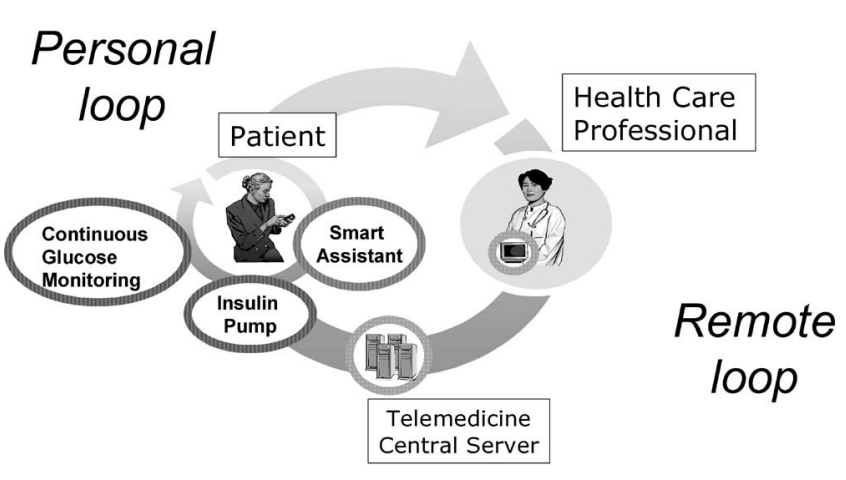

Fig. 1. INCA concept: Telemedical artificial pancreas.

control of the insulin pump based on glucose sensor data.

The four control strategies allow coping with specific patient preferences and requirements. The selection of a specific strategy, or combination of strategies, will depend on the different metabolic control situations of patients and also on the degree of patient's compliance. For example, an automatic personal loop control algorithm could be active at night, and a patient or a doctor control mode could be used during the day.

\section{System Design AND SPECIFICATION}

The design process of the INCA system is supported by a user-centered and model-based design method. The resulting concept and implementation of the personal loop, the remote loop, and their interaction processes intensively make use of current standards and a component-based approach to ensure its sustainability.

\section{A. MOSAIK-M INCA System Model}

The analysis and design of the INCA system is based on the previous experience of the research consortium [13], [15]. This knowledge and input from analysis and design phases of the project have been formalized using modeling, simulation, and animation of information and communication systems in medicine (MOSAIK-M) [20]. In INCA, the MOSAIK-M has been used for system analysis, system design, and costbenefit analysis. MOSAIK-M supports health information systems (HIS) analysis and design. It defines a generic process scheme and a meta model, and includes a tool environment. The generic process scheme guides modeling projects to produce HIS models of high quality in terms of correctness, completeness, and validity. The meta model defines the modeling language, and thus, the components of MOSAIK-M models, i.e., organizational structures (especially technical and human actors), processes, object and information structures, and software applications. MOSAIK-M models can be simulated and animated to support the analysis of HIS or user-oriented evaluation of HIS. Physiological models are not subject of the MOSAIK-M approach, but can be integrated in a MOSAIK-M model.

Two MOSAIK-M models have been created during the INCA project: an "as is" and a "to be"-model. The "as is"-model describes the current situation of a diabetic's treatment with an 
insulin pump, i.e., the problem domain. It identifies potential users of the system, basic organizational structures, processes and care-flows to support, and the definition and sources of medical data. The "as is"-model has been verified concerning its correctness and completeness due to the scope of the project by experts of the application field (diabetologists and diabetics), and was the starting point for generating a model of the INCA telemedical system [20].

The resulting "to be" INCA-model includes a comprehensive specification of the INCA system. It identifies 12 institutions and 37 roles of persons related to the INCA system; it describes 341 health care and technical processes that are directly or implicitly affected by the INCA system, and defines 81 application object classes and 358 application models. Thus, the "to be"-model is a blue print for implementing and introducing the INCA system into health care [20]. User interface prototypes are part of the INCA-model, and were used for a formative evaluation with diabetics, physicians, and technical partners prior to implementing the system. Their integration into the simulation of the system was further used to clear linguistic problems with the interpretation of user interfaces, which was very important in the international multilingual context of the project.

\section{B. Specification of the Personal Loop}

The SA mainly supports the personal loop and permits patients to perform the following tasks.

1) Management of monitoring data obtained from medical devices (pump, blood glucose meter and continuous glucose monitors) or directly from the patient (diet data and additional events affecting blood glucose profiles such as illness, menstruation, etc.).

2) Visualization of graphics and statistics of monitoring data.

3) Consultation of patient's active therapy in terms of basal profiles, bolus, and prescribed diet.

4) Virtual interface of the medical devices that allows patients to visualize and modify the configuration of their devices and to download monitoring data from them.

5) Data communication with/to the TMCS: to exchange text messages with the health care professionals (by current mail applications installed in the PDA), to synchronize the SA database, and to allow insulin pump remote control.

The other components that comprise the personal loop are the insulin pump, the continuous glucose monitoring system, and the blood glucose meter.

1) Insulin Pump: The insulin pump that was integrated into the personal loop was based on a D-TRON (Disetronic Medical Systems AG, Burgdorf, Switzerland). The pump is online reprogrammed according to the basal profile defined at the SA without the need of any manual operation on the pump.

The modified D-TRON insulin pump hardware is inherently safe. In case of an error, it is always detected, signalized, and handled, so that potentially hazardous situations can be prevented. In the remote control mode via a cordless connection to the PDA-based SA, the patient has to accept the insulin regime change (basal insulin as well as insulin boli), before the pump is reprogrammed.
2) Continuous Glucose Monitoring System: The original design of the SA is flexible in order to support the communication to any glucose sensor system.

The prototype for monitoring continuous glucose uses a microdialysis method with a bidirectional communication between the SA and the sensor test system. The communication protocol has been specified and implemented. However, the current specifications of this glucose sensor for being used in ambulatory environments prevented its use in clinical trials, and it obliged to find an alternative solution for a real-time continuous glucose monitoring.

The solution comes from an agreement with MedtronicMinimed to use the CGMS continuous glucose monitoring system that gives the physician the retrospective information about patient monitoring and the Medtronic-Minimed GuardianRT system that provides patients with real-time glucose readings. In both cases, continuous glucose profiles can be downloaded to the TMCS to be remotely available for patients and physicians. However, the bidirectional communication between the SA and the Medtronic sensors have not been specified, and therefore, neither implemented.

3) Glucose Meter: During the clinical trials, patients used the Accu-Chek Sensor Complete Meter (Roche Diagnostics $\mathrm{GmbH}$, Germany) to measure blood glucose readings. The system sends data to the telemedicine central server using a smart modem. The user interface for data transmission is the glucose meter and a modem activated with a unique button. Blood glucose data is afterwards available at the SA personal logbook.

\section{Specification of the Remote Loop}

The remote loop is supported by the TMCS. This telemedicine platform offers to both patients and physicians a tool for data management and visualization, and supports the communication with all user terminals.

The main Web features for patients are as follows: logbook data management; graphical reports of blood glucose values, continuous glucose monitoring data, and continuous insulin infusion data (i.e., line charts, pie charts, and $24 \mathrm{~h}$ charts); view therapy data; electronic health record management; messaging tools to create, send and manage electronic mails and online questionnaires; selecting and filling in questionnaires for technical, usable, and clinical evaluation of the system.

The main Web features for physicians are the following: Therapy management for creating and editing therapy recommendations; electronic health record management; view patient's logbook data; visualization of patient's monitoring data (blood glucose values, insulin values, diet, ketonuria, stress, illness, etc.); graphical reports of blood glucose values, continuous glucose monitoring data, and continuous insulin infusion data; messaging tools to create, send, and manage electronic mails; and follow-up of the questionnaires filled in by patients.

\section{Remote and Personal Loop Interaction Process Modeling}

In this section, we describe the interaction diagrams between INCA users and main components of the personal and remote loop systems. Fig. 2 presents an example of the sequence of actions within the personal loop. 


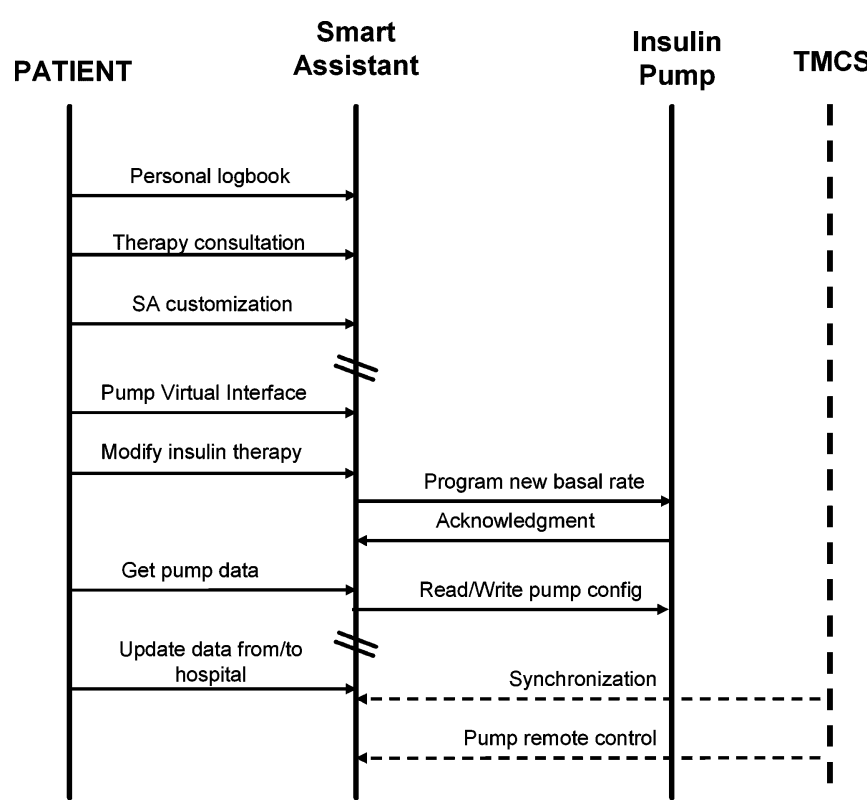

Fig. 2. Personal loop interaction diagram (TMCS: telemedicine central server).

The patient uses the SA as a personal logbook for disease selfmanagement, data monitoring analysis, communication with the medical devices, manual data entry, and consultation of current therapy proposed by physicians.

The SA pump virtual interface (PVI) allows for gathering information about the current pump programming and the insulin delivered over time (insulin basal rates and bolus). The PVI scenario enables patients to control the pump. New basal rates and current pump configuration, such as date and time, can be changed. After any programming operation, a confirmation is received in the PVI. For the closed-loop control, the SA is equipped with a control algorithm [12] that calculates insulin infusion rates upon patient's request.

The patient initiates a synchronization process to update personal monitoring data to the hospital or to download therapy changes recommended by the physician and other data stored at the server. This procedure sends the data stored in the SA to the INCA TMCS or retrieve new data from the TCMS to the SA.

Fig. 3 shows the interaction diagram of the remote loop process to support the physician decision process. The physician monitors the patient's metabolic state through the interaction with the TMCS Web application. He/she can propose a change in the insulin therapy that is notified to the patient, and is later on downloaded into the SA.

The physician could also directly manage the patient insulin pump through the pump remote control. The process involves a real-time notification to the SA that will be received by the patient. After an explicit patient consent, the SA reprograms the current insulin profiles in the pump.

\section{INCA SMART ASSISTANT}

\section{A. General Description}

The INCA SA communicates with the patient devices through a personal wireless network (local loop) and additionally

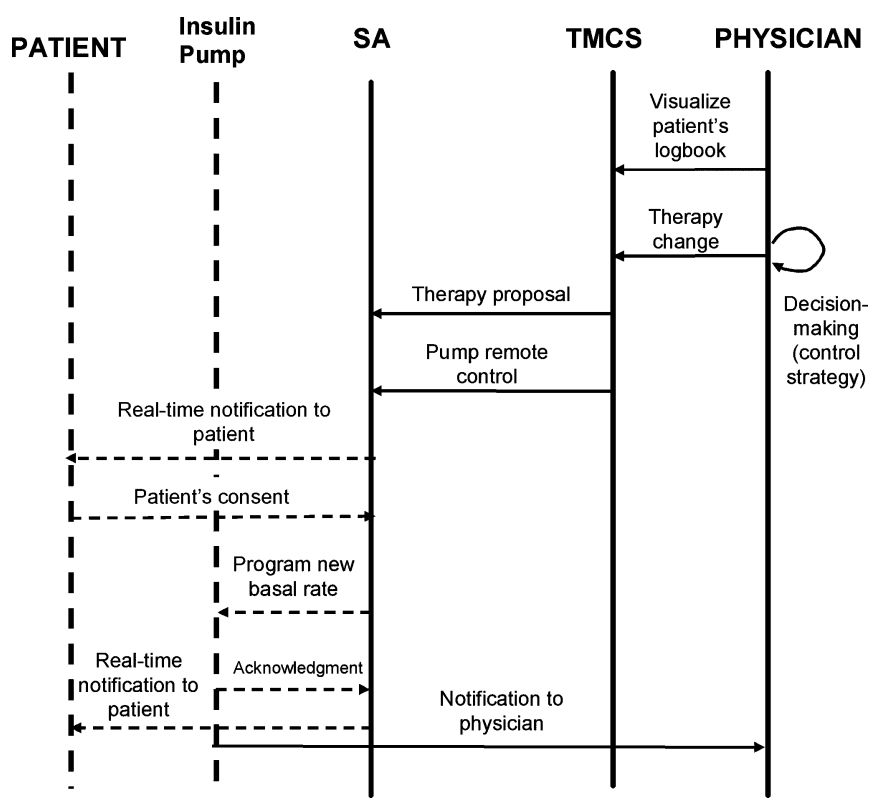

Fig. 3. Remote loop interaction diagram (SA: smart assistant; TMCS: telemedicine central server).

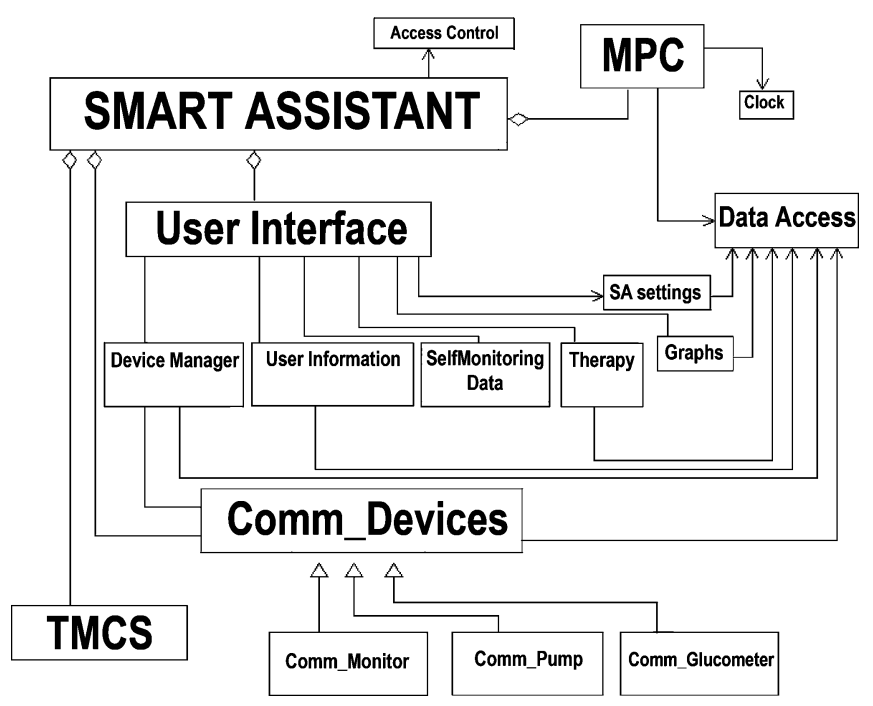

Fig. 4. SA software modules.

through a mobile wide area network (WAN) for providing telemonitoring, telecare, and remote information services (remote loop). The user terminal that supports the SA is a commercial PDA provided with wireless communication facilities, such as infrared, Bluetooth, and mobile GPRS. The SA works as a stand-alone system, supported on its own local application and database. The use of always-on networks such as the mobile GPRS, allows for a periodic update of the SA data from the medical devices and transfer new data to the INCA central server. The communication can be activated upon user's request.

\section{B. Smart Assistant Architecture}

The SA architecture consists of several software modules as shown in Fig. 4. 

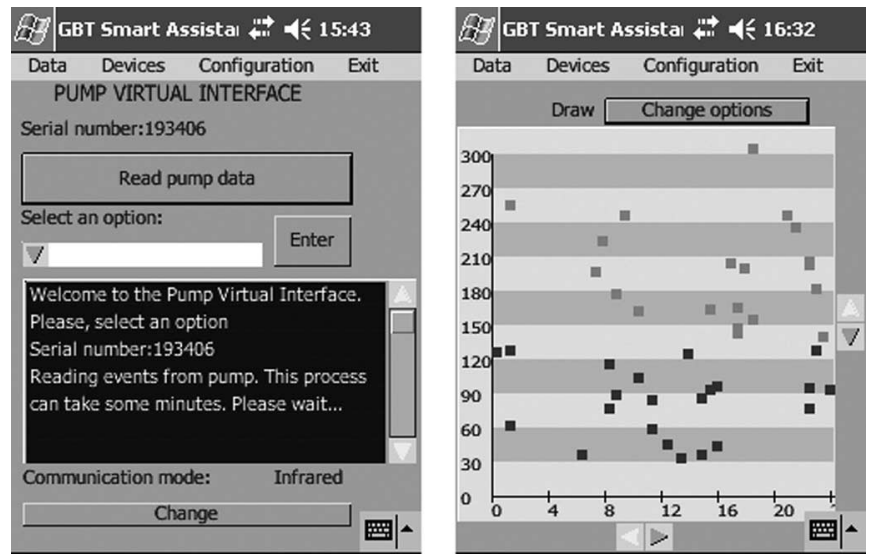

Fig. 5. Example of the SA user interface. (a) Pump virtual interface. (b) Graphic representation of blood glucose readings.

1) User Interface Module: The SA user interface supports multilanguage features, and was used in English, Spanish, Catalan, and German versions. The interface implementation is based on the Java Abstract Windows Toolkit (AWT), and offers a high scalability to the application.

Several submodules can be distinguished: the "DeviceManager" module provides a high-level vendor-independent interface for common procedures of reading and programming events on medical devices [see Fig. 5(a)]; the "User Information" module allows introducing personal data and the required password to access the database interchange process; the "Self MonitoringData" module allows data visualization in the patient logbook, and makes possible the introduction of new monitoring data and changes in previously entered data [see Fig. 5(b)]; the "Therapy" module allows the visualization of current insulin and diet treatment; The "SA_Settings" module sets user preferences, and registers changes in personal data.

2) Data Access Module: The Data_Access module provides all the other modules with the access to the SA local database using a Java Database Connectivity (JDBC) interface, both for data retrieval, modification and insertion.

3) Comm_Devices Module: The Comm_Devices manages the communications with the medical devices in the personal loop (the continuous glucose monitor, the glucose strip meter, and the insulin pump), supported via serial ports, infrared, and/or Bluetooth.

4) Model Predictive Control (MPC) Module: The MPC module implements the closed-loop model predictive control developed within the ADICOL experience [12]. The control algorithm is based on the physiologic model described by Hovorka et al. [21]. The MPC calculates changes in the insulin delivered by the pump based on results from the continuous glucose measurement. The algorithm can also provide insulin bolus advice before each meal intake. Using this algorithm within the SA implies extracting continuous monitoring data stored in the local database following the structure imposed by the MPC model.

5) Telemedicine Central Server Module: The TMCS module manages the interaction between the TMCS and the SA for data exchange as well as for the remote control of the medical devices. It requires an active mobile GPRS connection. The remote control procedures are supported on Common Object Request Broker Architecture (CORBA), which allows the SA to behave as an always on server. The SA receives external remote control requests via mobile GPRS [22].

\section{Hardware and Software Platform}

The SA hardware platform is an iPAQ hp2210 PDA that integrates Bluetooth for wireless communication, and it is provided with the AudioVox RTM 8000 for mobile GPRS communication capabilities. The software application is developed in a Java programming language using the Java Development Kit 1.1.8. and the CrEme v3.24 JVM that provides packages such as JDBC, CORBA, and RMI.

\section{TElemedicine CENTRAL SERVER}

\section{A. Telemedicine Architecture}

The architecture of the TMCS is based on a telemedicine multiaccess server (MAS) [23] that allows users to access the information, using any access terminal or combination of terminals they choose. The new features and software agents integrated in the architecture support the integration of the new devices and data considered in the INCA system: insulin pump, SA, and continuous glucose monitor.

A concept and a prototype system have been developed for the integration of the TMCS into a comprehensive regional health information infrastructure (RHII). The INCA diabetes documentation thus becomes a part of the patient's virtual shared health record. The INCA database is mounted with a connector/mediator module into the RHII, allowing authorized care providers to access: 1) information of the diabetes logbook and 2) the clinical diabetes documentation like laboratory results and findings. The information of the various interorganizational data sources is linked with unique patient identifiers stored on patient cards. For authorization purposes, a patient issues electronic tickets (so-called $e$-consents) to care providers. These e-consents are digitally signed certificates, authorizing the respective health professional for access to the shared health record for a limited time with individually parameterized access rights. Semantic interoperability is achieved by using the clinical document architecture (CDA) [24].

\section{B. Web-Based User Interface}

The starting point of the Web user interface was an existing telemedicine system [13], [15], [23] that has been modified to support the continuous monitoring data for diabetes control considered in the INCA system.

Fig. 6 shows an example of the Web-based user interface to allow patients and doctors to access through the Web to the continuous glucose monitoring and continuous insulin infusion data, enhancement of integrated data presentation, and printing of patient logbook.

\section{EVAluATION OF THE INCA SYSTEM}

The four control strategies of the INCA concept have been technically evaluated in the laboratory with prototype devices, 


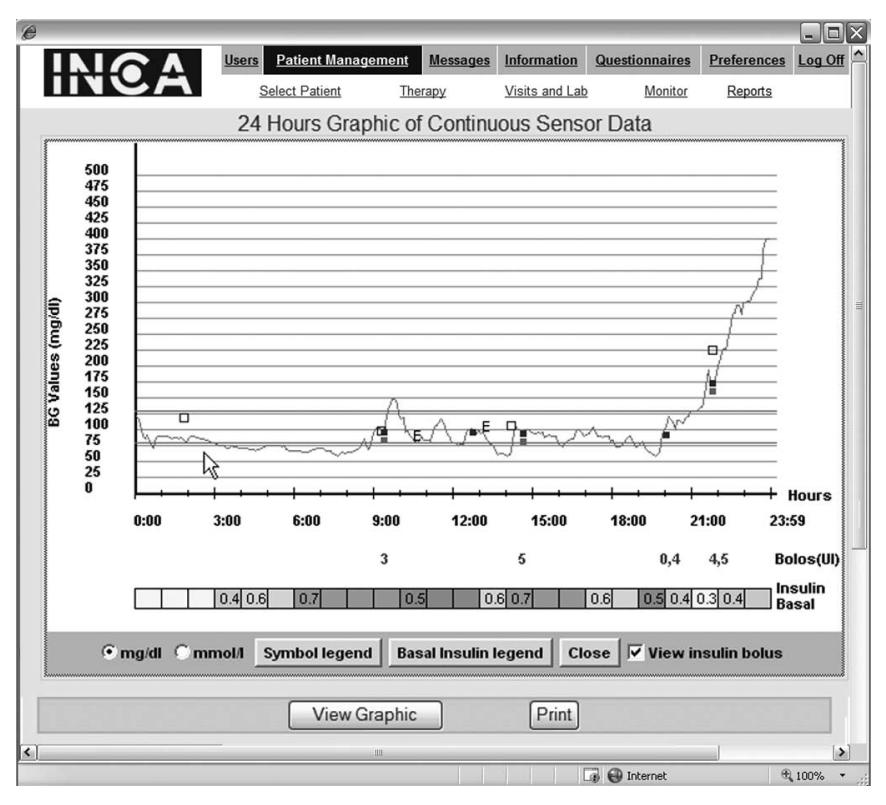

Fig. 6. Example of INCA Web-based user interface: patient report showing continuous glucose monitoring and continuous insulin infusion data.

but the clinical evaluation of strategies \#3 and \#4 is still unfinished due to the difficulty of using sensors and insulin pumps with real-time reading and programming in clinical pilots.

Up to date, we performed three clinical experiments [26], [27], and their results provided useful information about difficulties in the use of the SA technology and the impact of continuous glucose monitoring on patients' metabolic control.

The system evaluation assesses the performance according to technical implementation, user satisfaction, clinical utility, clinical efficacy and effectiveness, and quality of care. The evaluation has been conceived as a subjective evaluation, based on users' questionnaires, and an objective evaluation based on clinical data registered by health care professionals and the automatic tracking of user's interactions with the Web or PDA to obtain information about the use of the system.

\section{A. Feasibility Pilot}

Objective: To assess the technical feasibility of the INCA system. Clinical evaluation was not done at this stage.

Methods: During six months, a group of four Type 1 pumptreated diabetic patients from the Institut für Diabetesforschung (Munich, Germany) evaluated the technical components of the system using the control strategies \#1 and \#2.

Results: The pattern of system usage varies between individuals, but all of them registered and sent data to the hospital on a regular basis. Main technical difficulties came from the mobile GPRS communication and the use of the PDA: 1) GPRS connection failed when very high amounts of data were transmitted (e.g., data from several months); 2) the limited GPRS connection coverage in some suburban areas; and 3) PDA battery consumption during data transmission.

Conclusions: The use of the SA is feasible in ambulatory scenarios.

\section{B. Clinical Trial 1}

Objective: To evaluate the clinical utility of the INCA control strategies \#1 and \#2 based on BG self- monitoring and continuous insulin monitoring.

Methods: Ten Type 1 pump-treated diabetic patients from Hospital de Sant Pau (Barcelona, Spain) participated in a randomized crossover study (four weeks + four weeks). Patients were recruited from those regularly attended in the outpatients' diabetic clinics of the Hospital de Sant Pau, a tertiary hospital with a reference population of about 400000 people. After randomization, patients were allocated in one of the two groups: control or intervention. During the control phase, patients did not receive any feedback from the medical center, but they had to send glucose data for a retrospective analysis. During the intervention phase, patients were able to use both the SA and all the telemedicine services. Data sent by patients were analyzed by the physician, and modifications of the basal rates and boli were advised in the following $24 \mathrm{~h}$. Within the last week of each period, the patients used a three-day subcutaneous blind glucose monitoring (Medtronic-Minimed CGMS) for a retrospective evaluation between periods. Fructosamine and $\mathrm{HbA1c}$ were registered at the start and end of each period. Questionnaires and system data were used to assess the user satisfaction and system usage.

Results: After the experimental phase, fructosamine decreased significantly (393 \pm 32 versus $366 \pm 25 \mathrm{umol} / \mathrm{L} ; p<$ $0.05)$ and $\mathrm{HbAlc}$ tended to decrease $(8.0 \pm 0.6$ versus 7.78 $\pm 0.6 ; p=0.073$ ), while no changes were observed during the control phase [26]. In terms of system utility, all the patients stated that the INCA system helped them to communicate with the physician in an effective way and increased the flexibility of therapy management. All the patients stated that the use of the system makes them feel more secure in the handling of diabetes, and six out of ten patients considered that the system helped them to reduce the number of acute problems related to their diabetes (hypoglycaemias, ketosis, etc.). Patients would recommend the INCA system for diabetes care ( $78 \%$ in total agreement and $22 \%$ in partial agreement), and considered that it is easy to learn how to use it, and it helped them to understand and analyze their monitoring data quickly and more effectively.

Conclusions: The usage of the wireless SA for remote treatment advising allows better glycaemic control in pump-treated Type 1 diabetic patients. Patients' satisfaction was very high.

\section{Clinical Trial 2}

Objective: To evaluate the clinical utility of the INCA control strategies \#1 and \#2 based on continuous insulin monitoring and real-time continuous glucose monitoring versus the same INCA usage and capillary BG self-monitoring.

Methods: The ten Type 1 pump-treated diabetic patients from Hospital de Sant Pau (Barcelona, Spain) participated in a randomized crossover study (four weeks + four weeks) [27]. The design of the control phase was similar to the intervention phase in clinical trial 1 . In the intervention phase, patients additionally wore the continuous glucose sensor Guardian (Medtronic MiniMed, Northridge, CA), three days per week. 
Results: The usage and usability evaluation results did not show many differences in comparison to the clinical trial 1. After the intervention phase, HbA1c decreased in nine out of ten patients, the mean decrease being $0.8 \%(p=0.007)$. No change was observed during the control phase. Although HbA1c improved significantly, the percentage of glucose readings below 70 did not increase. Furthermore, patients did not suffer from any severe hypoglycaemia along the study.

Conclusions: The experiment demonstrated that patients achieved a better metabolic control when using real-time glucose monitoring enhanced with the INCA control strategies \#1 and \#2.

\section{Cost-Benefit Analysis}

Cost-benefit analyses of systems like INCA are needed to prepare the decision on their introduction for routine care. To conduct a cost analysis on the example of Germany, the MOSAIK$M$ "as is"-model and the "to be"-model were parameterized with cost values from the German KoDiM study, and a period of one year was simulated to determine the yearly costs of diabetes management and treatment for a patient who does not suffer from diabetes-related complications yet [28]. The HbA1c-value was chosen as an effectiveness parameter for diabetes therapy. To determine the probability of developing complications and their probable duration, the Archimedes model [29] was used. It was parameterized with selected HbA1c-values anticipating the effect of INCA, respectively, from the trials. The yearly costs of conventional insulin pump treatment for a 19-year-old diabetes Type 1 patient with no complications are $5907 €$ (German health care system; September 2005). Using the INCA system with continuous blood glucose measurement of $24 \mathrm{~h}$ a day and seven days a week would raise the yearly costs by $7348 €$.

The cost efficiency of the INCA approach highly depends on the cost of the continuous blood glucose measurement. Further work is needed to complete the cost-benefit analysis by including calculating monetary effects on treatment costs due to a reduced $\mathrm{HbA} 1 \mathrm{c}$-value as determined by the clinical studies, and indirect (e.g., loss of working hours) and intangible (e.g., reduction of quality of life) costs.

\section{DISCUSSION}

The enormous biomedical engineering efforts performed over the last three decades still leave room for improvement in order to achieve a "technological solution" for diabetes: an artificial pancreas system to be used by diabetic patients at any time and place. This challenge implies new research work to face yet unsolved difficulties: 1) insulin pumps that allow real-time insulin infusion programming are not commercially available; 2 ) the need of a reliable and cost-efficient continuous glucose monitoring technology; 3) the development and clinical validation of safe and efficient closed-loop control algorithms, coping with the inherent complexity of an SC-SC setup in ambulatory environments; and 4) the integration of these new technologies and therapeutic procedures within a telemedicine system that provides patients and doctors a safe, convenient, and efficient tool in a day-by-day normal life.
The INCA concept has defined four closed-loop control strategies in which the decision-making process moves from the physician to the patient and from the patient to an automatic decision-support system. The INCA technology is ready for implementing in clinical routine to all these approaches. However, the current stage of glucose sensing and insulin infusion technologies allowed the clinical evaluation of the first and second control strategies in which a complete automatic telemedical closed-loop solution is not considered.

The use of insulin pumps in an artificial pancreas requires a periodic and automatic adjustment of the insulin dosage depending on the glycaemic values. In order to do this, a real-time programmable pump must be utilized. In order to demonstrate the technical feasibility, we used a modified insulin pump provided by the partner Disetronic Medical Systems AG. This modified pump is remotely controllable (basal insulin as well as insulin boli) within the INCA system via a cordless connection to the PDA-based SA. History data can be downloaded from the pump via Bluetooth and infrared communication to the SA, which afterwards can be transmitted to the TMSC.

Another requirement for implementing a telemedical artificial pancreas is the acquisition of glucose measurements with minimum delays in real time. Several technologies and approaches have been applied to implement real-time continuous glucose sensors. The most widely investigated type of glucose sensor is the amperometric enzyme electrode implanted in the SC tissue [4] such as the Guardian RT (Medtronic) that shows glucose data in real time every $5 \mathrm{~min}$. Other companies such as DexCom and Abbott follow the same technological approach for their amperometric needle-type sensors. However, some inherent problems of this technology are the insufficient signal stability of the glucose electrodes and the accuracy limitations in situations of hypoglycaemia and hyperglycaemia. Microdialysis as used in the Menarini system, although frequently related with technical signal delays, generally avoids undesirable sensor-tissue interactions.

The SA has been designed in a flexible way so that different sensor systems can be supported and practically used. Due to its high robustness, our first choice was therefore to use a microdialysis-based system with the Sa. A prototype of such a sensor provided real time glucose data to the SA that was entered into a closed-loop control algorithm [12] that was also implemented in the SA. Laboratory and technical feasibility tests of the personal loop were done with this sensor. However, the lack of suitable microdialysis sensor systems to be used in ambulatory settings did not allow any testing in the clinical trials. In order to implement the INCA SA in a telemedical clinical setting, we decided to employ the Guardian RT (Medtronic). This sensor was integrated in the telemedicine applications and allowed us to leverage the benefits of continuous glucose monitoring and telemedicine, but the sensor did not permit to test the MPC closed-loop algorithm because sensor data could not be downloaded in real time to the SA. Current research focuses on integrating the new sensor devices in the SA architecture.

The INCA architecture has been designed to follow the expected rapid evolution of mobile technologies as all the longdistance communications assume the availability of any IP 
network that are also available in the new mobile $3 \mathrm{G}$ networks. However, this standardized scenario is not so mature in the personal area network where medical devices have to be connected and operated. The main issue is that communications with insulin pumps, continuous glucose sensors, and glucometers are not standardized at all and the integration of any new device into the SA will require new developments and the collaboration of manufactures to replicate the communication protocols.

Telemedicine has demonstrated its efficiency to decrease HbA1c of patients [13], [1], and the research presented here extends the good outcomes of telecare together with the continuous glucose monitoring to achieve the artificial pancreas in real-life settings.

\section{CONCLUSION}

The concept and realization of a mobile telemedicine closedloop system for diabetes management has been presented in this paper. The INCA system implemented for the first time a PDA-based SA for patients that manage a continuous glucose sensor and an insulin pump, integrated in a telemedicine system that offers to diabetic patients and doctors a Web-based access for continuous glucose monitoring and continuous insulin infusion data. The results of the clinical trials show the technical feasibility of the telemedical artificial pancreas concept and the clinical benefits of the use of continuous glucose monitoring system within a personal assistant, integrated in a telemedicine system, to better the HbA1c of patients. The ultimate goal of this research is to build a telemedical artificial pancreas toward looking for new technological solutions to improve the quality of care and quality of life of people with diabetes.

\section{ACKNOWLEDGMENT}

The authors would like to acknowledge all researchers of the INCA consortium, especially to V. Torralba (Bioing and Telemedicine Center, Universidad Politécnica de Madrid), I. Hoffmann (Institut F. Medizinische Informatik, Braunschweig, Germany), T. Kaupper (Diabetes Research Centre, Munich, Germany), as well as to I. Hutzli and M. Haeberli. They would also like to thank Medtronic Company (Madrid, Spain) for the provision of the continuous glucose monitoring systems (Guardian RT).

\section{REFERENCES}

[1] World Health Organization (2006, May 30) Fact Sheet $N^{\circ} 138$ [Online]. Available: http://www.who.int/mediacentre/factsheets/fs138/en/

[2] The Diabetes Control and Complications Trial Research Group, "The effect of intensive treatment of diabetes on the development and progression of long-term complications in insulin-dependent diabetes mellitus," N. Engl. J. Med., vol. 329, pp. 977-986, 1993.

[3] E. R. Carson and C. Cobelli, Eds., Modelling Methodology for Physiology and Medicine. San Diego, CA: Academic Press, 2001.

[4] E. J. Gomez, M. E. Hernando, and F. del Pozo, "Biomedical and information technologies for diabetes care," Wiley Encyclopedia Biomed. Eng., vol. 2, pp. 1064-1078, 2006.

[5] S. Andreassen, E. J. Gomez, and E. R. Carson, "Computers in diabetes 2000," Comput. Methods Programs Biomed., vol. 69, no. 2, pp. 93-95, 2002.

[6] R. S. Parker, F. J. Doyle,III, and N. A. Peppas, "The intravenous route to blood glucose control," IEEE Eng. Med. Biol. Mag., vol. 20, no. 1, pp. 65-73, Jan. 2001.
[7] R. Bellazzi, G. Nucci, and C. Cobelli, "The subcutaneous route to insulin-dependent diabetes therapy," IEEE Eng. Med. Biol. Mag., vol. 20, no. 1, pp. 54-64, Jan./Feb. 2001.

[8] R. Hovorka, "Continuous glucose monitoring and closed-loop systems," Diabet. Med., vol. 23, pp. 1-12, 2005.

[9] B. W. Bequette, "A critical assessment of algorithms and challenges in the development of a closed-loop artificial pancreas," Diabetes Technol. Ther, vol. 7, no. 1, pp. 28-47, 2005.

[10] C. Owens, H. Zisser, L. Jovanovic, B. Srinivasan, D. Bonvin, and J. Doyle, III, "Run-to-run control of blood glucose concentrations for people with type 1 diabetes mellitus," IEEE Trans. Biomed. Eng., vol. 53, no. 6, pp. 996-1005, Jun. 2006.

[11] R. Dudde, T. Vering, G. Piechotta, and R. Hintsche, "Computer-aided continuous drug infusion: setup and test of a mobile closed-loop system for the continuous automated infusion of insulin," IEEE Trans. Inf. Technol. Biomed., vol. 10, no. 2, pp. 395-402, Apr. 2006.

[12] R. Hovorka, L. J. Chassin, M. E. Wilinska, V. Canonico, J. A. Akwi, M. O. Federici, M. Massi-Benedetti, I. Hutzli, and C. Zaugg, et al., "Closing the loop: the adicol experience," Diabetes Technol. Ther, vol. 6, no. 3, pp. 307-318, 2004.

[13] E. J. Gómez, M. E. Hernando, A. Garcia, F. del Pozo, R. Corcoy, E. Brugués, J. Cermeño, and A. de Leiva, "Telemedicine as a tool for intensive management of diabetes: The DIABTel experience," Comput. Meth. Prog. Biomed., vol. 69, pp. 163-177, 2002.

[14] J. Wojcicki, P. Ladyzynski, J. Krzymien, E. Jozwicka, J. Blachowicz, E. Janczewska, K. Czajkowski, and W. Karnafel, "What can we really expect from telemedicine in intensive diabetes treatment: Results from 3-year study on type 1 pregnant women," Diabetes Technol. Ther, vol. 3, pp. 581-589, Dec. 2001.

[15] R. Bellazzi, C. Larizza, S. Montani, A. Riva, M. Stefanelli, G. D’ Annunzio, R. Lorini, E. J. Gomez, M. E. Hernando, and E. Brugués, "A telemedicine support for diabetes management: the T-IDDM project," Comput. Methods Programs Biomed., vol. 69, no. 2, pp. 147-161, 2002.

[16] K. E. Smith, B. A. Levine, S. C. Clement, M. J. Hu, A. Alaoui, and S. K. Mun, "Impact of mycareteam for poorly controlled diabetes mellitus," Diabetes Technol. Ther., vol. 6, no. 6, pp. 828-835, 2004.

[17] C. Larizza, R. Bellazzi, M. Stefanelli, P. Ferrari, P. De Cata, C. Gazzaruso, P. Fratino, G. D'Annunzio, M. E. Hernando, and E. J. Gómez, “The M2DM Project-The experience of two Italian clinical sites with clinical evaluation of a multi-access service for the management of diabetes mellitus patients," Methods Inf. Med., vol. 45, no. 1, pp. 79-84, 2006.

[18] P. M. Trief, P. C. Morin, R. Izquierdo, J. A. Teresi, J. P. Eimicke, R. Goland, J. Starren, S. Shea, and R. S. Weinstock, "Depression and glycaemic control in elderly ethnically diverse patients with diabetes: The IDEATel project," Diabetes Care, vol. 29, no. 4, pp. 830-835, 2006.

[19] I. Zimmer-Galler and R. Zeimer, "Rresults of implementation of the digiscope for diabetic retinopathy assessment in the primary care environment," Telemed. e-Health, vol. 12, no. 2, pp. 89-98, 2006.

[20] O. J. Bott, J. Bergmann, I. Hoffmann, T. Vering, E. J. Gómez, M. E. Hernando, and D. P. Pretschner, "Analysis and specification of telemedical systems using modelling and simulation: The MOSAIK-M approach," Stud. Health Technol. Inf., vol. 116, pp. 503-508, 2005.

[21] R. Hovorka, F. Shojaee-Moradie, P. V. Carroll, L. J. Chassin, I. J. Gowrie, N. C. Jackson, R. S. Tudor, A. M. Umpleby, and R. H. Jones, "Partitioning glucose distribution/transport, disposal, and endogenous production during IVGTT," Am. J. Physiol. Endocrinol. Metab., vol. 282, no. 5, 2002.

[22] M. E. Hernando, E. J. Gómez, A. Gili, M. Gómez, G. García, and F. del Pozo, "A mobile intelligent personal assistant for telemedical closed-loop strategies in diabetes," in Proc. MEDICON04:"2nd Health Telematic Mediterranean Conf. Med. Biol. Eng., Napoli, Italy, 2004, pp. 145-155.

[23] M. E. Hernando, G. García, E. J. Gómez, and F. del Pozo, "Intelligent alarms integrated in a multi-agent architecture for diabetes management," Trans. Inst. Meas. Control, vol. 26, no. 3, pp. 185-200, 2004.

[24] J. Bergmann, O. J. Bott, D. P. Pretschner, and R. Haux, "An econsent-based shared EHR system architecture for integrated healthcare networks," Int. J. Med. Inf., vol. 76, no. 2-3, pp. 130-136, 2007.

[25] R. H. Dolin, L. Alschuler, S. Boyer, C. Beebe, F. M. Behlen, P. V. Biron, and A. Shabo Shvo, "HL7 clinical document architecture, release 2," $J$. Am. Med. Inf. Assoc., vol. 13, pp. 30-39, 2006.

[26] M. Rigla, M. E. Hernando, E. J. Gómez, E. Brugués, V. Torralba, G. García-Sáez, J. Vilaverde, A. de Leiva, "Asynchronic remote advice through a wireless personal assistant improves glycaemic control in pump treated type 1 diabetic patients," presented at the 5th Annu. Diabetes Technol. Meet., San Francisco, CA, Nov. 2005. 
[27] M. Rigla, M. E. Hernando, E. J. Gómez, E. Brugués, V. Torralba, G. García-Sáez, R. Cabezas, and A. de Leiva, "Glycaemic control improvement with the weekly use of the GuardianRT together with a wireless assistant for asynchronic remote advice in pump treated type 1 diabetic patients," Diabetologia, vol. 49, pp. 153-154, 2006.

[28] O. J. Bott, I. Hoffmann, J. Bergmann, P. Kosche, C. von Ahn, D. C. Mattfeld, O. Schnell, and D. P. Pretschner, "Simulation based cost-benefit analysis of a telemedical system for closed-loop insulin pump therapy of diabetes," Stud. Health Technol. Inf., vol. 124, pp. 435-441, 2006.

[29] L. Schlesinger and D. M. Eddy, "Archimedes: A new model for simulating health care systems-The mathematical formulation," J. Biomed. Inf., vol. 35, pp. 37-50, 2002.

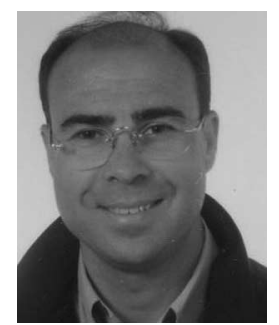

Enrique J. Gómez (S'83-M'85-SM'06) is currently a Professor of biomedical engineering at the Universidad Politécnica de Madrid, Madrid, Spain. He is the author or coauthor of more than 260 papers published in various scientific journal papers and conference proceedings in related fields, and has directed 39 national and international research projects. His current research interests include telemedical closedloop control systems for diabetes management and medical imaging visualization and integration for virtual surgery simulation and planning.

Prof. Gómez is an Associate Editor of the IEEE TRANSACTIONS ON INFORMATION TECHNOLOGY IN BIOMEDICINE (TITB). He is also the Treasurer of the European Alliance for Medical and Biological Engineering and Sciences (EAMBES) Society and the Secretary General of the Spanish Society for Biomedical Engineering (SEIB).

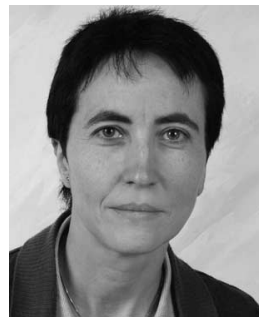

Mercedes Rigla Cros received the M.D. and Ph.D. degree.

Since 1997, he has been with the Endocrinology and Nutrition Department, Hospital Sant Pau, Barcelona, Spain. She is a specialist in endocrinology and nutrition trained from the University of Barcelona and Autonomous University of Barcelona. Her current research interests include diabetes mellitus, particularly technology applied to diabetes. She is the author or coauthor of 26 original publications and 9 chapters related to endocrinology and diabetes. She has collaborated in activities of the DiabCare, DiabCard, M2DM, and the Advanced Insulin Infusion using a Control Loop (ADICOL) European Projects.

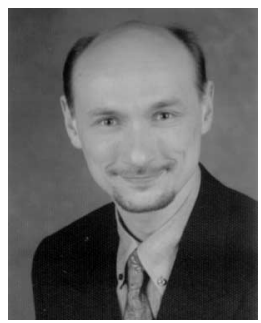

Oliver Bott received the Ph.D. degree in computer science in 2001 from the Technical University of Braunschweig, Braunschweig, Germany.

$\mathrm{He}$ is currently a Scientific Assistant at the Institute for Medical Informatics, Technical University Braunschweig. His current research interests include methods and tools for analysis, design and management of health information systems and transinstitutional health information system architectures for cooperative, patient centered, and shared care.

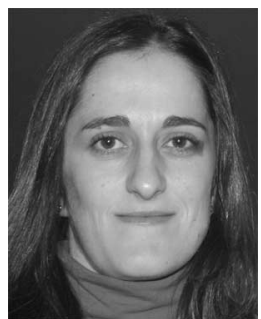

Gema García-Sáez received the Telecomunications Eng. degree in 2003 from the Universidad Politécnica de Madrid, Madrid, Spain.

She is currently a Researcher at the Biomedical Engineering and Telemedicine Department, Universidad Politécnica de Madrid. Her current research interests include telemedicine systems, knowledge management, and data analysis for diabetic patients. She has experience in distributed systems and mobile computing.

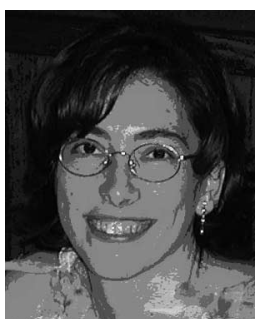

M. Elena Hernando Pérez (M'01) received the Telecommunication Eng. degree in 1990 and the $\mathrm{Ph} . \mathrm{D}$. degree in 1997, both from the Universidad Politécnica of Madrid (UPM), Madrid, Spain.

She is currently an Associate Professor at UPM. Her main expertise is in the field of telemedicine services for chronic patients and the definition of decision support tools for diabetes care. Her current research interests include the development of an intelligent telemedical artificial-pancreas system combining continuous glucose monitoring and insulin pumps controlled by closed-loop algorithms.

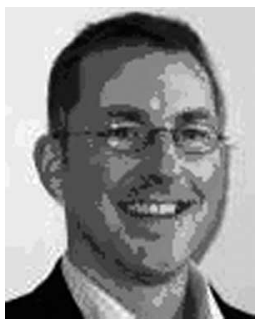

Thomas Vering received the Ph.D. (Summa cum laude) degree from the Technical University Munich, Munich, Germany.

He was with the Institute for Chemo- and Biosensors, Muenster, Germany. He is currently with the Disetronic Medical Systems AG, Burgdorf, Switzerland. He was a Coordinator and Originator of the EC projects such as the Advanced Insulin Infusion using a Control Loop (ADICOL), INCA, and the Closed Loop Insulin Infusion for Critically Ill $\mathrm{Pa}$ tients (CLINICIP). After the integration of Disetronic into Roche Diagnostics Diabetes Care in 2003, he held the position of the Head of the Technology Program "Therapeutic Advice." Since April 2007, he is the Program Head for Tight Glycemic Control of Roche Professional Diagnostics.
Peter Pretschner, photograph and biography not available at the time of publication.

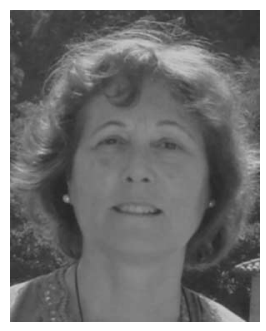

Eulalia Brugués received the Graduate degree in pharmacy and the Master's degree in information system technologies.

She is currently with the Endocrinology Department, Hospital Sant Pau, Barcelona, Spain. She is the coinvestigator in many EC projects related to diabetes care and information technology.

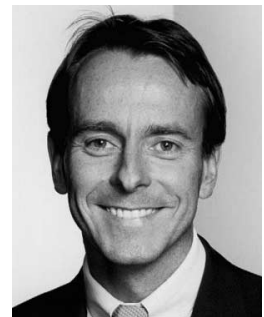

Oliver Schnell is currently a Professor of Medicine at the Ludwig-Maximilians-University, Munich, Germany and the Head of the Department of Cardiovascular Disease, Diabetes Research Institute, Munich. During 1983-1989, he was at the University of Saarland, Saarland, Germany, Harvard Medical School, Boston, MA, and University of California, San Francisco. His current research interests include diabetes and vascular diseases, cardiac disease in diabetes, immunology, interventions and new treatment strategies, and telemedicine and new media.

Prof. Schnell is a Member of the Guideline Committee of the American Association of Clinical Endocrinologists (AACE) and the Program Committees of National and International Scientific Conferences. He is Editor-in-Chief of the "Diabetes, Metabolism and the Heart" journal. 
Caroline Patte was in Paris, France, in 1979. She received the Technical Biomedical Eng. degree from the University of Technology in Compiègne, France.

In 2003, she joins Disetronic Medical Systems AG, Burgdorf, Switzerland, where she is currently the Study Manager for two clinical studies as well as a Project Leader of the EC funded Project Closed Loop Insulin Infusion for Critically Ill Patients (CLINICIP). She is also engaged in Roche Diabetes Care in the Research and Technology on the INCA Project.

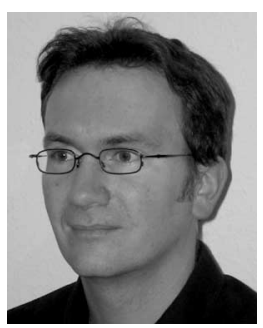

Joachim Bergmann received the Diploma from the University of Hildesheim, Hildesheim, Germany, and the Ph.D. degree from the Technical University of Braunschweig, Braunschweig, Germany, both in computer science.

$\mathrm{He}$ is currently a Researcher in the Institute for Medical Informatics, Technical University of Braunschweig. His current research interests include architectures of transinstitutional health information systems and integration of electronic patient records.

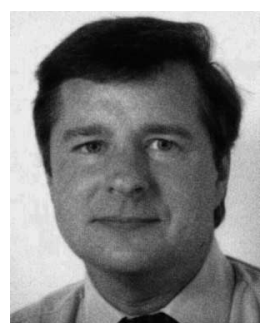

Ralf Dudde received the Ph.D. degree in physics from the University of Hamburg, Hamburg, Germany, in 1989.

He was a Postdoctoral Researcher at the IBM Research Laboratory, Rüschlikon, Switzerland. In 1990, he joined the Fraunhofer Institute of Silicon Technology, Itzehoe, Germany, where he is currently the Head of the Department of Product Development. He has initiated and coordinated several international research projects as a project manager for microsystem sensor and microelectronic development projects.

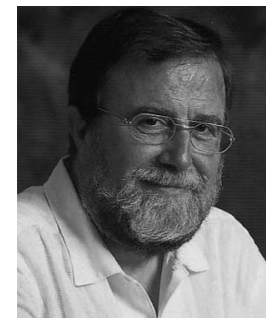

Alberto de Leiva received the Master's degree in health economics from the University Pompeu i Fabra, Barcelona, Spain, the Medical Doctor degree from the University of Barcelona, Barcelona, and the $\mathrm{Ph} . \mathrm{D}$. degree in medical sciences with specialization in physiology from the University of Madrid, Madrid, Spain.

He was a Resident in Internal Medicine and Endocrinology, University of Madrid and a Research Fellow of the American College of Endocrinology. He is currently a Professor of medicine, Univ Autònoma, Barcelona (UAB) and the Director of the Institute of Research and Department of Endocrinology and Diabetes, Hospital Sant Pau-UAB. He is the author or coauthor of more than 250 scientific articles published in various international journals.

Prof. Leiva is the Vice president of the European Association for the Study of Diadetes (EASD), the President of the Deutsche Pfadfinderschaft Sankt Georg (DPSG), and the International Association of the Diabetes and Pregnancy Study Groups (IADPSG). He is the recipient of the International Clinician Award from the American College of Endocrinology and the Jorgen Pedersen Medal (DPSG-EASD). 